\title{
Magnetic Clouds: Solar Cycle Dependence, Sources, and Geomagnetic Impacts
}

\author{
Y. Li $^{1}$ - J.G. Luhmann' ${ }^{1}$ B.J. Lynch ${ }^{1}$
}

Received: 23 April 2018 / Accepted: 21 September 2018 / Published online: 2 October 2018

(C) The Author(s) 2018

\begin{abstract}
Magnetic clouds (MCs) are transient magnetic structures giving the strongest southward magnetic field (Bz south) in the solar wind. The sheath regions of MCs may also carry a southward magnetic field. The southward magnetic field is responsible for spaceweather disturbances. We report a comprehensive analysis of $\mathrm{MCs}$ and $\mathrm{Bz}$ components in their sheath regions for 1995 to 2017. 85\% of 303 MCs contain a south Bz up to $50 \mathrm{nT}$. Sheath Bz during the 23 years may reach as high as $40 \mathrm{nT}$. MCs of the strongest magnetic magnitude and Bz south occur in the declining phase of the solar cycle. Bipolar MCs depend on the solar cycle in their polarity, but not in the occurrence frequency. Unipolar MCs show solar-cycle dependence in their occurrence frequency, but not in their polarity. MCs with the highest speeds, the largest total- $B$ magnitudes, and sheath $\mathrm{Bz}$ south originate from source regions closer to the solar disk center. About $80 \%$ of large Dst storms are caused by MC events. Combinations of a south $\mathrm{Bz}$ in the sheath and south-first MCs in close succession have caused the largest storms. The solar-cycle dependence of bipolar MCs is extended to 2017 and now spans 42 years. We find that the bipolar MC Bz polarity solar-cycle dependence is given by MCs that originated from quiescent filaments in decayed active regions and a group of weak MCs of unclear sources, while the polarity of bipolar MCs with activeregion flares always has a mixed Bz polarity without solar-cycle dependence and is therefore the least predictable for $\mathrm{Bz}$ forecasting.
\end{abstract}

Keywords Coronal mass ejection · Magnetic clouds

Electronic supplementary material The online version of this article (https://doi.org/10.1007/s11207-018-1356-8) contains supplementary material, which is available to authorized users.

Y. Li

yanli@ssl.berkeley.edu

1 Space Sciences Laboratory, University of California at Berkeley, 7 Gauss Way, Berkeley, CA 94804, USA 


\section{Introduction}

Coronal mass ejections (CMEs) may carry an enhanced magnetic field and fast-moving plasma into the heliosphere. The magnetic field of a CME originates in the low solar atmosphere, where a stressed magnetic structure becomes unstable and erupts, escaping the Sun. Interplanetary CMEs (ICMEs) that exhibit an in-situ topology of helical magnetic flux ropes in interplanetary magnetic field (IMF) observations are referred to as magnetic clouds (MCs) (e.g. Burlaga, 1988; Bothmer and Schwenn, 1998). Herein, we use the Mulligan, Russell, and Luhmann (1998) classifications that describe the behavior of the $\mathrm{Bz}$ component within $\mathrm{MC}$ flux ropes as corresponding to axis orientations with low inclination and high inclination with respect to the ecliptic plane as bipolar and unipolar MCs, respectively. When a bipolar MC passes the observer, the IMF time series often shows a large component (and rotation) of the $\mathrm{Bz}$ within the $\mathrm{MC}$, which can be either $\mathrm{S}$ (southward) in the leading portion and $\mathrm{N}$ (northward) in the trailing portion, defined as an SN MC, or the reverse, defined as a NS MC. Unipolar MCs generally have only a $\mathrm{S}$ or $\mathrm{N} \mathrm{Bz}$ component, referred to as S MCs or N MCs. Thus the magnetic field of MCs may present four different types: SN, NS, S, and N. MCs often carry a long-lasting steady southward Bz field, making them important for space-weather concerns (e.g. Zhang et al. 2004, 2007; Gopalswamy et al., 2008; Richardson and Cane, 2013). Predicting the Bz south field has been a challenging task and attracted coordinated efforts (Riley, 2016; Riley and Love, 2017). The Bz component of the interplanetary field plays an important role in the coupling of the solar wind and IMF with the magnetosphere of the Earth. MCs also compress and disturb the ambient solar wind and IMF to form sheath regions ahead. Fast MCs often drive shocks, and the sheath regions between the shock and the magnetic flux rope can have an enhanced $\mathrm{Bz}$ component.

The Bz fields within MCs have been studied by many authors, while Bz fields in their sheath regions have not been studied systematically. We obtain the sheath southward $\mathrm{Bz}$ for 23 years starting from 1995 in this article. The Bz polarity in bipolar MCs has been found to show a dependence on the solar cycle (Zhang and Burlaga, 1988; Bothmer and Rust, 1997; Bothmer and Schwenn, 1998; Mulligan, Russell, and Luhmann, 1998). Li et al. $(2011,2014)$ extended these results, showing that the Bz in bipolar MCs has a cyclic reversal on the timescale of the solar magnetic cycle over three sunspot cycles. It has been reported that about a third of the ICMEs are MCs (Gosling, 1990). The ratio between MCs and ICMEs depends on the solar cycle, and almost all ICMEs are MCs near solar minimum, but the proportion of MC ICMEs near solar maximum is much lower (Richardson and Cane, 2010). During Solar Cycle 23, approximately $48 \%$ of ICMEs are MCs, and $40 \%$ of MCs are bipolar MCs (Li et al., 2011, 2014). The speed of the bipolar MCs has essentially the same distribution as all ICMEs, which implies that they are not from any special group of CMEs in terms of the solar origin. Although CME flux ropes may undergo a number of changes during the eruption and propagation processes or be sampled in situ with a variety of impact parameters, a significant number of MCs evidently retain sufficient similarity to the orientation of their source-region magnetic field to possess the same cyclic periodicity in polarity reversal. During solar minima, the Bz field at the leading portion of a bipolar MC is the same as the solar global dipole field (also noted by Mulligan, Russell, and Luhmann, 1998). This finding suggests that MCs preferentially remove the like polarity of the solar dipole field, thereby supporting the idea that CMEs play a role in the solar magnetic cycle. The solar-cycle dependence of MC orientation is interesting not only because it shows the connection between the MC magnetic field and the solar magnetic field, but also because, as mentioned above, the large and long-lasting $\mathrm{Bz}$ field component has important spaceweather implications. 
Since 2007, the twin Solar Terrestrial Relations Observatory (STEREO) spacecraft have been orbiting the Sun at about 1 AU near the ecliptic plane in addition to the Advanced Composition Explorer (ACE) and Wind spacecraft at the $\mathrm{L}_{1}$ point, making three independent observing points of the in-situ solar wind and IMF near the ecliptic plane at $1 \mathrm{AU}$ during an extended period for the first time. Moreover, STEREO images offer stereoscopic views of the corona and CMEs, which are extremely useful in accurately identifying the source regions of CMEs.

In this article, after a brief description of the data source and events selection (Section 2), we present a comprehensive study of MCs encountered at the $\mathrm{L}_{1}$ point from 1995 to 2017, when we have continuous high-quality data. We analyze both bipolar and unipolar MCs to investigate the solar-cycle dependence of the Bz field, as well as the south Bz component in the sheath region ahead of the MCs (Section 3). We then identify the solar sources for MCs at $\mathrm{L}_{1}$ from 2007 to 2017, when multiple views of the Sun are available after the launch of STEREO. We separate bipolar MCs into groups according to the types of their source regions and examine the solar-cycle dependence of the Bz field for each group (Section 4.1). We also identify and divide the solar sources into groups according to the types of MCs they give rise to and present their locations in solar coordinates and against the background of the magnetic butterfly diagram (Section 4.2). We then study the association of the MCs with geomagnetic storms measured by the Dst index (Section 5). Finally, we update the MC polarity solar-cycle dependence by extending the study through 2017 using bipolar MCs encountered both at the $\mathrm{L}_{1}$ point and the STEREO twin spacecraft sites (Section 6). We conclude the article with discussions and conclusions regarding the overall $\mathrm{MC}$ characteristics and their implications (Section 7).

\section{MC Event Selections}

We used solar-wind plasma and IMF in-situ measurements on ACE at the $\mathrm{L}_{1}$ point and on the STEREO twin spacecraft orbiting the Sun at $\approx 1$ AU (treating the observations on each spacecraft as an independent dataset). We analyzed five-minute ACE magnetic field (MAG) and Solar Wind Electron, Proton, and Alpha Monitor (SWEAPAM) merged level-2 data, and STEREO-A and -B ten-minute in-situ measurements of particles and cme transients (IMPACT) and Plasma and suprathermal ion composition (PLASTIC) merged level-2 data. By examining the three components of the IMF and the solar-wind plasma parameters of proton temperature, density, bulk speed, and the plasma beta, we selected MC intervals. To identify MCs, we required i) an enhanced magnetic-field magnitude greater than $\approx 8 \mathrm{nT}$, ii) a low-variance magnetic field with a coherent rotation of the field vector over a time interval on the order of a day, and iii) a lower-than-average proton temperature (e.g. Burlaga, 1988). The selection process was made by visual inspection, in the same manner as previous studies for consistency (Mulligan, Russell, and Luhmann, 1998; Li et al., 2011, 2014), except that we lowered the requirement for magnetic enhancement to be no less than $10 \mathrm{nT}$ to no less than $8 \mathrm{nT}$ because some good MC events can be missed, especially in Solar Cycle 24, when the magnetic field is weaker (e.g. see Lee et al., 2009; Kilpua et al., 2014).

Note that in this study we do not include structures that can be classified as ICMEs, but do not have a clearly identifiable MC driver (e.g. see Jian et al., 2006). As discussed by Jian et al. (2006) and other authors, the general consensus is that MCs are generally present in ICMEs as drivers but are best observed when a spacecraft or observer is more centrally located within the passing structure. Thus some of our results mainly apply to the more 
centrally sampled ICMEs. In addition, the initial flux ropes in CMEs can be distorted during their propagation, e.g. as a result of interactions between CMEs (Lugaz et al., 2017).

With these criteria, we have identified $303 \mathrm{MCs}$ at $\mathrm{L}_{1}$ using $\mathrm{ACE}$ and/or OMNI data (omniweb.gsfc.nasa.gov/ow.html) from 1995 to 2017, including 194 bipolar MCs and 109 unipolar MCs. All of the MCs at $\mathrm{L}_{1}$ point and their sheath southward Bz field, as well as their source regions, are studied and presented in the next section. We have identified 67 bipolar MCs at STEREO-A and -B from 2007 to 2013 due to data availability and continuity issues in and after 2014. We have also identified eight bipolar MCs at STEREO-A in 2016 and 2017. Bipolar MCs from all three spacecraft are used to extend the record of their polarity, long-term variation, and dependence on the solar cycle at the end of the article.

\section{Magnetic Field in MCs and Sheath Regions}

Since 1995 , the in-situ data at $\mathrm{L}_{1}$ have good quality and essentially continuous coverage. We found 194 bipolar MCs and 109 unipolar MCs for the 23 years from 1995 to 2017, including $103 \mathrm{SN}, 91 \mathrm{NS}, 64 \mathrm{~S}$, and $45 \mathrm{~N}$ MCs. We note that there are only 45 north-only (N type) MCs out of the total $303 \mathrm{MCs}$, which means that $258 \mathrm{MCs}$ contain a south $\mathrm{Bz}$ in their internal field, i.e. $85 \%$ of MCs contain a south Bz component in part or for the entire duration of the MC passage over the Earth, clearly showing the importance of MCs for geo-disturbances.

Figure 1a presents the annual counts of NS and SN bipolar MCs versus time, Figure 1b has the annual counts of the $\mathrm{S}$ and $\mathrm{N}$ unipolar MCs versus time, Figure 1c shows the annual counts of bipolar MCs as positive values and unipolar MCs as negative values, Figure 1d gives the annual counts of total MCs in an open histogram and those MCs with a south Bz component in a filled histogram, Figure 1e shows the normalized polarity histogram based on the annual counts in Figure 1a, and Figure 1f shows the sunspot numbers of Cycles 23 and 24. The vertical lines in light-gray mark the two solar minima in 1996 and 2009, and the dark-gray lines mark the two solar maxima in 2000 and 2014. The polarity of the bipolar MCs has a clear solar-cycle dependence, as was known previously. The polarity of the unipolar MCs, however, has no such trend. The occurrence of unipolar MCs is much less frequent around solar minima. The number of bipolar MCs is greater than that of unipolar MCs as a whole and also in each year except in 2001. The occurrence of MCs (Figure 1d) does not have clear solar-cycle dependence, while the occurrence of general ICMEs does, as reported previously (Jian et al., 2006; Jian, Russell, and Luhmann, 2011; Richardson and Cane, 2010). Note that this only holds for the occurrences; the south magnetic-field magnitude within MCs is shown next.

In addition to the plasma and magnetic-field properties within MCs, the magnetic-field strength and polarity in the ICME sheath regions ahead of MC ejecta are also important parameters in terms of the space-weather effects of these events. In Figure 2, we present these parameters versus time: (a) maximum ICME plasma speed, (b) maximum magneticfield magnitude within MCs, (c) maximum value of Bz south within MCs, (d) maximum value of Bz south in the MC sheath region, and (e) the sunspot numbers from 1995 to 2017 spanning Cycles 23 and 24. Again, the light- and dark-gray lines mark the solar minimum and maximum, respectively. The red and blue circles represent parameters for SN and NS bipolar MCs, respectively. The black and green plus symbols are for $\mathrm{S}$ and $\mathrm{N}$ unipolar MCs, respectively. The red, blue, black, and green squares represent parameters of the sheath regions ahead of SN, NS, S, and N MCs, respectively. In Solar Cycle 23 (24), the MC speeds range from 300 to 1000 (700) $\mathrm{km} \mathrm{s}^{-1}$, the magnetic magnitudes within MCs range from 8 to 62 (40) nT, the south magnetic fields within MCs range from 0 to 45 (22) nT, and the 
Figure 1 (a) Annual counts of bipolar MC NS and SN MCs; (b) annual counts of unipolar $\mathrm{N}$ and S MCs; (c) annual counts of bipolar and unipolar MCs;

(d) annual counts of MCs (open bars) and the MCs that contain south Bz fields (filled bars); (e) normalized polarity histogram based on the annual counts in Figure 1a; (f) sunspot numbers of Solar Cycles 23 and 24.

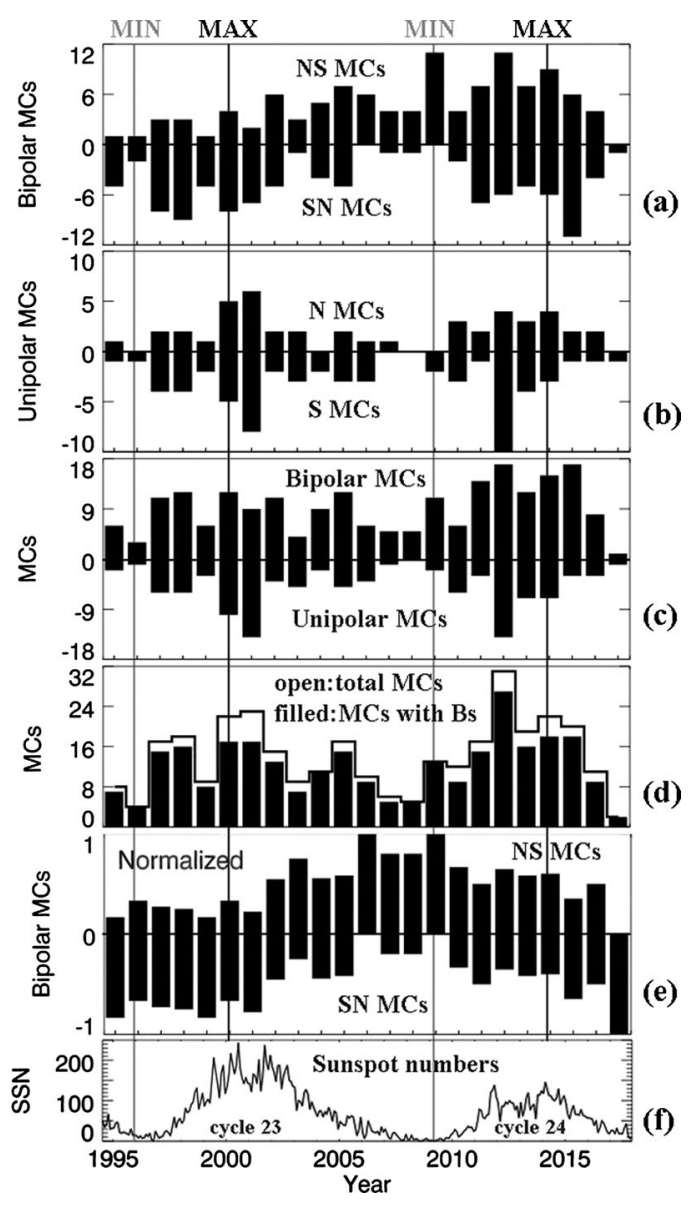

south magnetic fields in the MC sheaths range from 0 to 42 (17) nT. The ICMEs are slower and the field strengths are much weaker in Cycle 24. In Cycle 23, the highest values of all parameters appear in the declining phase of the solar cycle between the solar maximum and the next solar minimum. Cycle 24 seems to have the same tendency, but it is less obvious, perhaps for two reasons: first, that the values are all less significant, and second, that the cycle is still not complete.

The south magnetic fields within MCs $\left[B_{\mathrm{s}}\right]$ and in the sheath regions $\left(B_{\mathrm{ss}}\right)$ are reported here for the two solar cycles, while we have previously shown corresponding results for speed and total magnetic magnitude of MC in Li et al. (2011). Figure 2 shows that the MC speed $[V]$ and the total magnetic-field magnitude $\left[B, B_{\mathrm{s}}\right.$, and $\left.B_{\mathrm{ss}}\right]$ are all solar-cycle dependent, being weaker around solar-activity minimum and stronger around solar-activity maximum and during a large part of the declining phase. The geoeffectiveness of the MC events and the combined effect of strong $B_{\mathrm{s}}$ and $B_{\mathrm{ss}}$ is discussed in Section 7.

In Figure 3, we present the MCs analyzed above versus ICMEs found by a few other studies. Figure 3a presents the MCs as a black line and ICMEs from three other research groups in gray lines of different shades (www.srl.caltech.edu/ACE/ASC/DATA/level3/icmetable2.htm, Richardson and Cane, 2010; space.ustc.edu.cn/dreams/wind_icmes/h, Chi et al., 2016; www-ssc.igpp.ucla.edu/ jlan/ACE/Level3/ICME_List_from_Lan_Jian.pdf, Jian et al., 2006, 


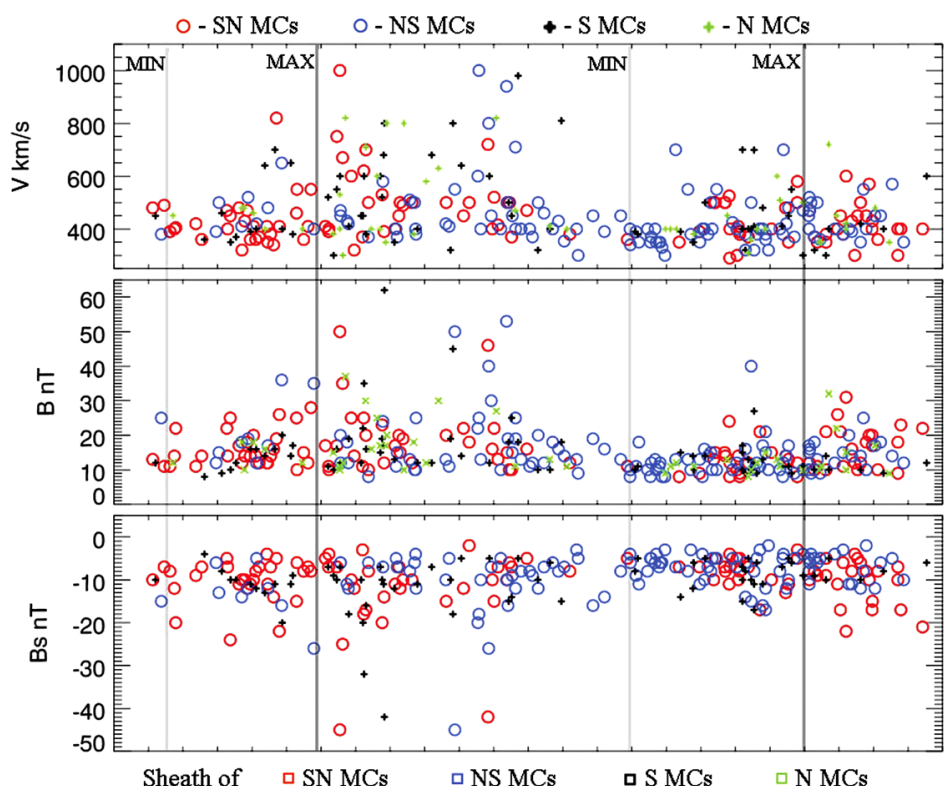

(a)

(b)
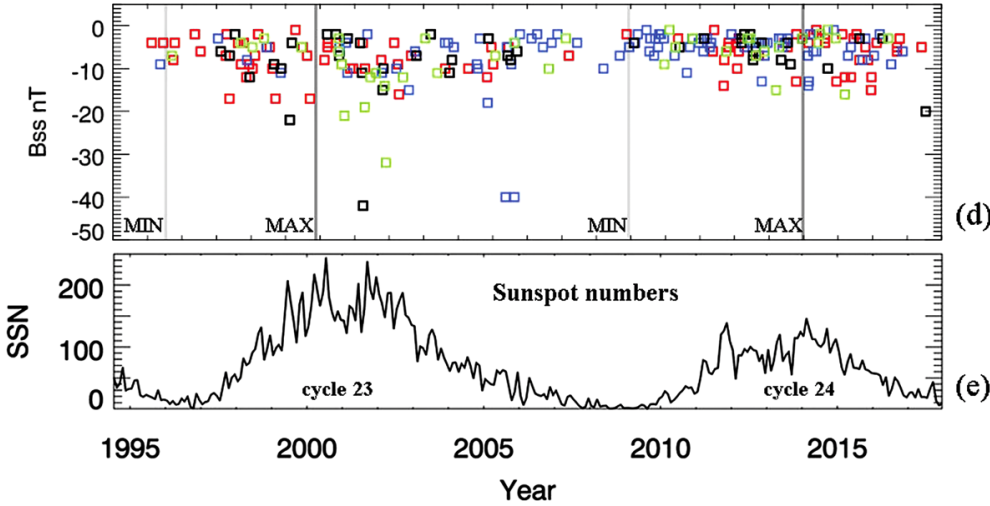

Figure 2 (a) MC plasma speed; (b) peak value of the magnetic-field magnitude within MCs; (c) peak value of $\mathrm{Bz}$ south within $\mathrm{MCs}$; (d) peak value of $\mathrm{Bz}$ south in the sheath region before MCs; and (e) the sunspot numbers for reference. The light-and dark-gray lines mark the solar minimum and maximum, respectively. The red and blue circles represent parameters of SN and NS MCs, respectively. The black and green plus sign are for S and N MCs, respectively. The red, blue, black, and green squares represent parameters of the sheath regions before SN, NS, S, and N MCs, respectively.

2013; Jian, Russell, and Luhmann, 2011), Figure 3b has the ratio of MCs versus ICMEs, and Figure 3c shows the sunspot numbers spanning Cycles 23 and 24 again as reference. The vertical lines in light-gray mark the two solar minima in 1996 and 2009, and those in dark-gray mark the two solar maxima in 2000 and 2014. The occurrence of MCs (Figure 3a) does not have a solar-cycle dependence, while the occurrence of ICMEs does, as previously reported (Jian et al., 2006; Jian, Russell, and Luhmann, 2011; Richardson and Cane, 2010). The ratio between MCs and ICMEs (Figure 3b) has a solar-cycle dependence with the maximum ratio as high as unity at solar minima, and as low as 0.3 at some point on the rising phase of the solar activity (using the sunspot numbers in Figure $3 \mathrm{c}$ for reference). 
Figure 3 (a) Time series of MC annual counts and ICME annul counts adapted from three other authors; (b) ratio of each year between MCs and ICMEs; (c) sunspot numbers of Solar Cycles 23 and 24.

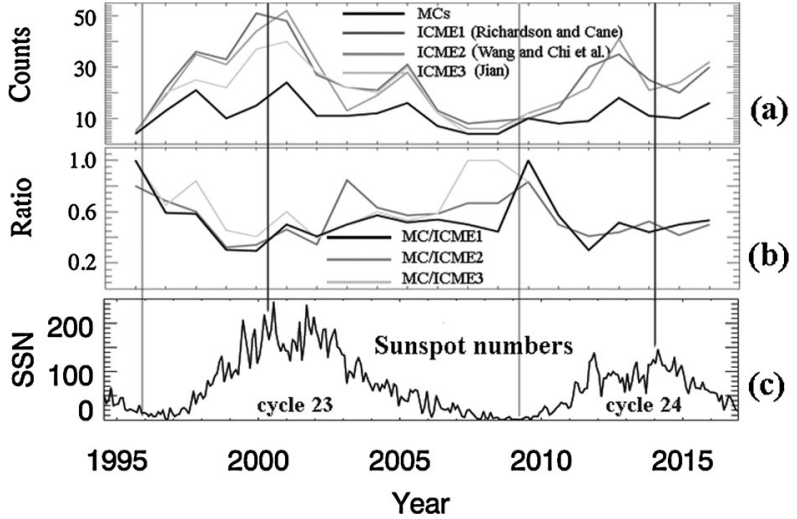

\section{MCs and Solar Sources}

Since 2007, when the STEREO twin spacecraft were launched, we have had multiperspective views of the Sun, as well as full-disk coronal images at higher cadence and with better availability than had previously been available. In 2010, the launch of the Solar Dynamics Observatory (SDO) provided the community with Earth-view full-disk images at higher cadence and resolution, and excellent quality. With these unprecedented resources, researchers are able to more confidently make the associations between in-situ MC events and their parent CMEs, including identifying the source regions of the CMEs in the lower solar atmosphere. In our identification of the solar sources of the $\mathrm{L}_{1} \mathrm{MCs}$, we utilized the images and movies from the Large Angle and Spectrometric Coronagraph (LASCO) CME catalog and STEREO Science Center, the SDO images and movies from the Sun in Time website sdowww.Imsal.com/suntoday_v2/index.html made available by the LockheedMartin Solar and Astrophysics Lab (LMSAL), and the Geostationary Operational Environmental Satellite (GOES) soft X-ray flare and other information from the Solar Monitor website (www.solarmonitor.org). We first searched the LASCO CME catalog for halo, partial halo, or wide CMEs greater than $60^{\circ}$ during the five days prior to the $\mathrm{MC}$ arrival. Often, there are multiple candidate sources for a CME during active periods of the Sun near solar maximum. In these cases, multi-perspective observations made possible with the STEREO twin spacecraft are particularly helpful. Multi-views are also extremely useful for identifying backside halo CMEs. STEREO coronagraph and Heliospheric Imagers (HI) images were examined whenever available for better certainty of the correspondence between a $\mathrm{CME}$ and the MC at the $\mathrm{L}_{1}$ point. We further identified the associated activity in the low corona, including solar flares, filament eruptions, and EUV dimmings (e.g. Thompson et al., 1998; Krista and Reinard, 2017). Clearly, there is an added step to identify sources for MCs than CMEs, and therefore more uncertainty is involved.

From 2007 to 2017, 149 MCs including 100 bipolar MCs and 49 unipolar MCs have been found that encountered the Earth. Forty-two MCs had active-region flare sources, and $57 \mathrm{MCs}$ had filament-eruption sources. For $8 \mathrm{MCs}$, only dimming was seen in EUV movies as the activity source, and one MC had a weak eruption-like signature in EUV movies as the source. Forty MCs had unclear sources. When an MC event is marked with a flare source, a GOES soft X-ray flare is recorded greater than $\approx \mathrm{B} 8.0$ from an active region, we took the flare over the other CME signatures present to classify the MC source. When an MC is marked with a filament-eruption source, the filament is a large one in a decayed active region. A large filament eruption sometimes may also have a soft X-ray flare. In this case, 
Figure 4 Bipolar MCs separated into groups according to different types of sources. Annual counts of NS and SN MCs that originated from active-region flares (a); large quiescent filaments (b); and unclear sources (c). The bottom panel shows the sunspot numbers of 2007 to 2017 (d).

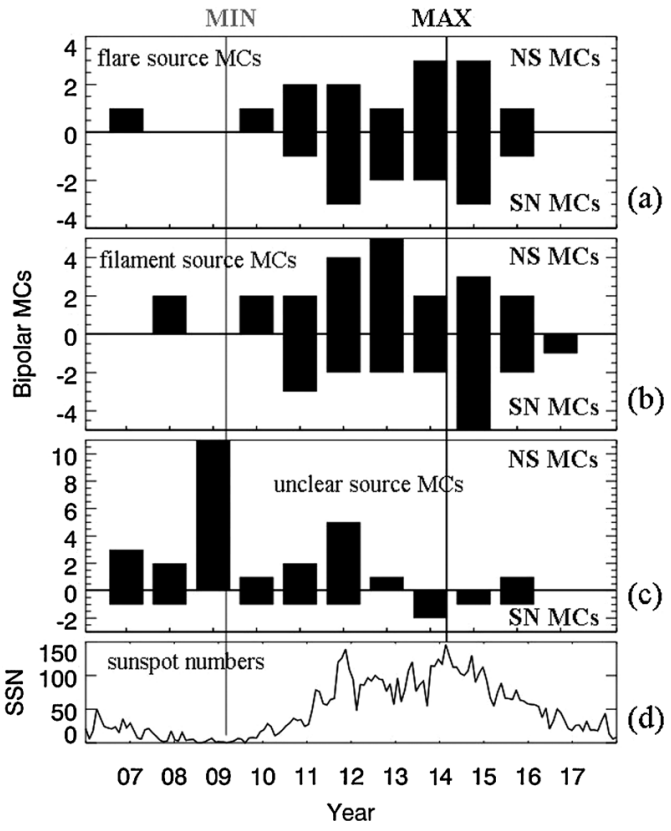

we took the filament over the flare to classify the MC source. When an MC was identified with a dimming source, the dimming in the EUV images or movies was the only signature associated with the CME event. The MCs with no clear source include these cases: i) the identified parent $\mathrm{CME}$ has no identifiable eruption signature at the low corona (11 out of $40 \mathrm{MCs}$ ); ii) no suitable coronagraph CME is identified in the LASCO CME catalog in our five-day window (13 out of $40 \mathrm{MCs}$ ); iii) uncertain parent CMEs: multiple possible source CMEs ( 8 out of $40 \mathrm{MCs}$ ), and iv) uncertain sources: multiple possible source activities (10 out of $40 \mathrm{MCs}$ ). The first case of $11 \mathrm{CMEs}$ corresponds to the so-called "stealth" CMEs (Robbrecht, Patsourakos, and Vourlidas, 2009; D'Huys et al., 2014; Alzate and Morgan, 2017).

\subsection{Bipolar MCs Grouped by Solar Source Type}

Of the 100 bipolar MCs, 27 had flare sources, 39 had filament sources, 27 had unclear sources, 6 had dimming sources, and 1 had a small eruption-like source. We include the first three types in Figure 4 as three separate bar plots to investigate their polarity variation with time. Figure 4 shows the polarity of bipolar MCs from (a) flare sources, (b) filament sources, and (c) unclear sources. Figure 4d shows sunspot numbers over the same time range. The flare-source MCs rarely occur in the period of low solar activity around solar minimum, but they occur much more frequently around solar maximum, as expected. No solar-cycle polarity trend exists for this group of bipolar MCs with flare sources, unlike what was described in the previous section for the set of all bipolar MCs. The bipolar MCs with filament sources do reflect the solar-cycle polarity trend. If one ignores the one SN outlier MC in 2011, the solar-cycle trend is better shown. Considering that the number of events is so small, we conclude that this group does show the same solar-cycle dependence with fluctuations. The MCs with unclear sources may also be considered as having the same solarcycle dependence. In fact, the NS MCs around the last solar minimum were mostly from the 


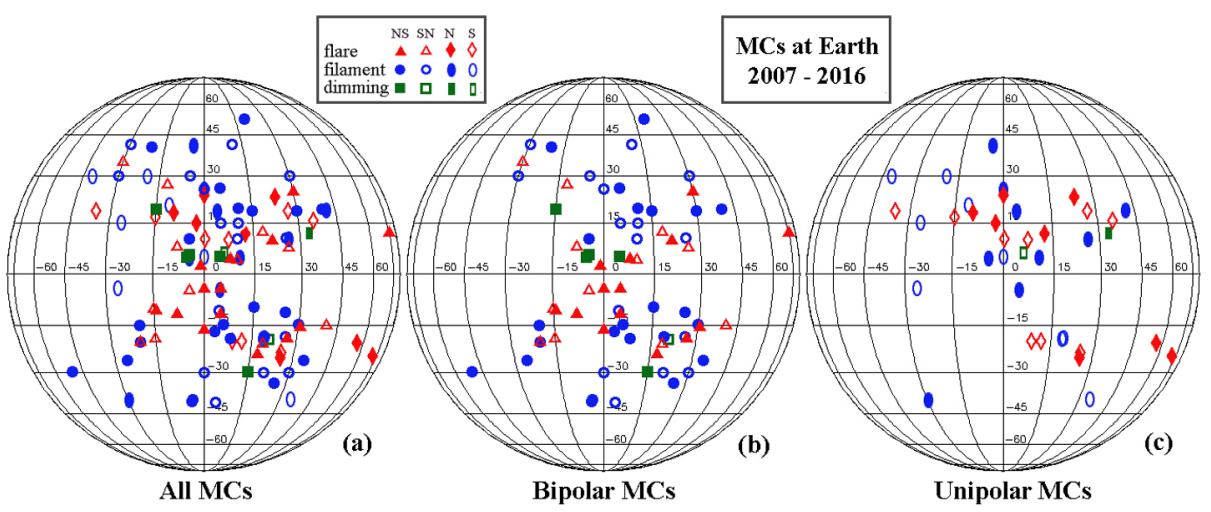

Figure 5 Locations of all types of MC source regions in the solar coordinate system. Specifically, (a) source regions for all MCs from 2007 to 2017 at $\mathrm{L}_{1}$; (b) source regions of bipolar MCs; (c) source regions of unipolar MCs. The symbols: filled-red triangles are for flares giving rise to NS MCs; open-red triangles are for flares giving rise to SN MCs; filled-red diamonds are flares for N MCs; open-red diamonds are flares for S MCs; filled-blue circles are filaments for NS MCs; open-blue circles are filaments for SN MCs; filled-blue ellipses are filaments for N MCs; open-blue ellipses are filaments for S MCs; and filled-green squares are EUV dimming for NS MCs.

contributions of the third group of weak MCs with unclear sources including stealth CME. Since stealth CMEs arise from coronal streamers (e.g. see Lynch et al., 2016, and references therein), the field directions of these MCs might be expected to agree with the solar dipole field.

Only by separating MCs into the three groups do we have a clear picture that the bipolar MC polarity solar-cycle dependence is given by those MCs that originated from quiescent filaments in decayed active regions and the group of weak MCs of unclear sources, including stealth CMEs.

\subsection{Solar Sources and Parameters}

Of the MCs (including both bipolar and unipolar) with identifiable sources, $42 \mathrm{MCs}$ had flare sources and $57 \mathrm{MCs}$ had filament eruption sources. For $8 \mathrm{MCs}$, only dimming signatures are observed in EUV images. We recorded the locations of these source regions, and we present them in Figure 5 in solar coordinates as seen from Earth, and in Figure 6 in synoptic format over the magnetic butterfly diagram up to 2016 by D. Hathaway at NASA (solarscience.msfc.nasa.gov/dynamo.shtml). In Figures 5 and 6, red symbols represent flares, blue symbols represent filaments, green symbols are for dimming cases, solid symbols are for north-first or north-only MCs, and open symbols are for south-first or southonly MCs; for further details, see figure annotations and captions. Figure 5a includes all events, Figure 5b includes bipolar MCs, and Figure 5c) includes unipolar MCs. Most of the sources are located within $45^{\circ}$ latitudes and longitudes with respect to the subsolar point (highlighted by orange lines), except for a few outliers. Flare sources in general are located at lower latitudes than large filament sources, which is reasonable considering the filament association with decayed active regions. More source regions are located in the western than in the eastern hemisphere (Hess and Zhang, 2017). The source regions do not appear to be distinguished or ordered by their resulting MC types in this format of display. Figure 6 includes the solar source locations of (a) all events, (b) bipolar events, and (c) unipolar events. From 2007 to about 2014, more source regions are seen in the northern hemisphere, but 

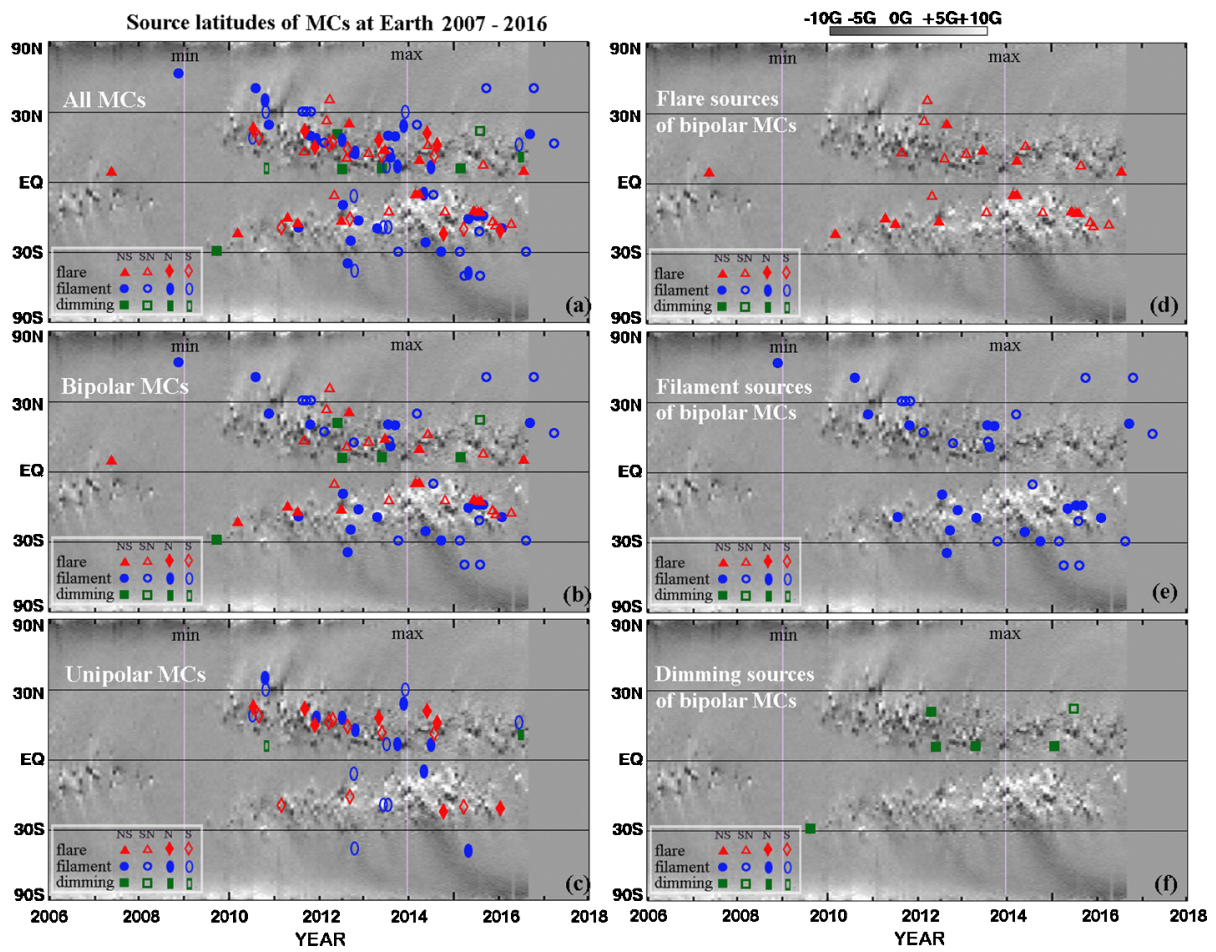

Figure 6 Locations of MC source regions overlaid on the magnetic butterfly diagram by D. Hathaway (NASA). The vertical lines (2009 and 2014) mark solar minimum and maximum. (a) Source regions for all MCs from 2007 to 2017 at $\mathrm{L}_{1}$ on the butterfly diagram; (b) source regions of bipolar MCs on the butterfly diagram; (c) source regions of unipolar MCs on the butterfly diagram; (d) flare sources of bipolar MCs; (e) large-filament sources of bipolar MCs; and (f) dimming sources of bipolar MCs. The symbols are the same as in Figure 5. The symbols: filled-red triangles are for flares giving rise to NS MCs; open-red triangles are for flares giving rise to SN MCs; filled-red diamonds are flares for N MCs; open-red diamonds are flares for S MCs; filled-blue circles are filaments for NS MCs; open-blue circles are filaments for SN MCs; filled-blue ellipses are filaments for N MCs; open-blue ellipses are filaments for S MCs; and filled-green squares are EUV dimming for NS MCs.

after 2014, more sources are seen in the southern hemisphere (Hess and Zhang, 2017). In Figures $6 \mathrm{~d}$, e, and $\mathrm{f}$, we present the bipolar MC subset of solar source locations for flare sources, filament sources, and dimming sources, respectively. The flare sources show the greatest agreement with the butterfly patterns formed by active regions, as expected. This format shows more clearly that the large filament sources are located at higher latitudes than flare sources as a whole. In Figure 6d, flare sources for NS MCs (filled-red triangles) and SN MCs (open-red triangles) are quite mixed and not ordered by time, as shown in Figure 4a in bar-plot form. In Figure 6e, filament sources for NS MCs (filled-blue circles) and SN MCs (open-blue circles) are separated. Here, the mostly filled-blue circles starting in 2007 switch to being mostly open-blue circles toward 2017, as shown in Figure $4 \mathrm{~b}$ in a bar plot. In Figure $6 f$, there are too few cases to show any well-defined trend, although filled-green squares (sources for NS MCs) occur first, with the last one in 2015 giving way to open-green squares (sources for SN MCs). These MC sources with only dimming signatures on the solar disk may correspond to stealth CMEs when the sources are on the disk instead of at the limb. If this is a correct interpretation, these events may be added to the group shown in Figure 4d, 

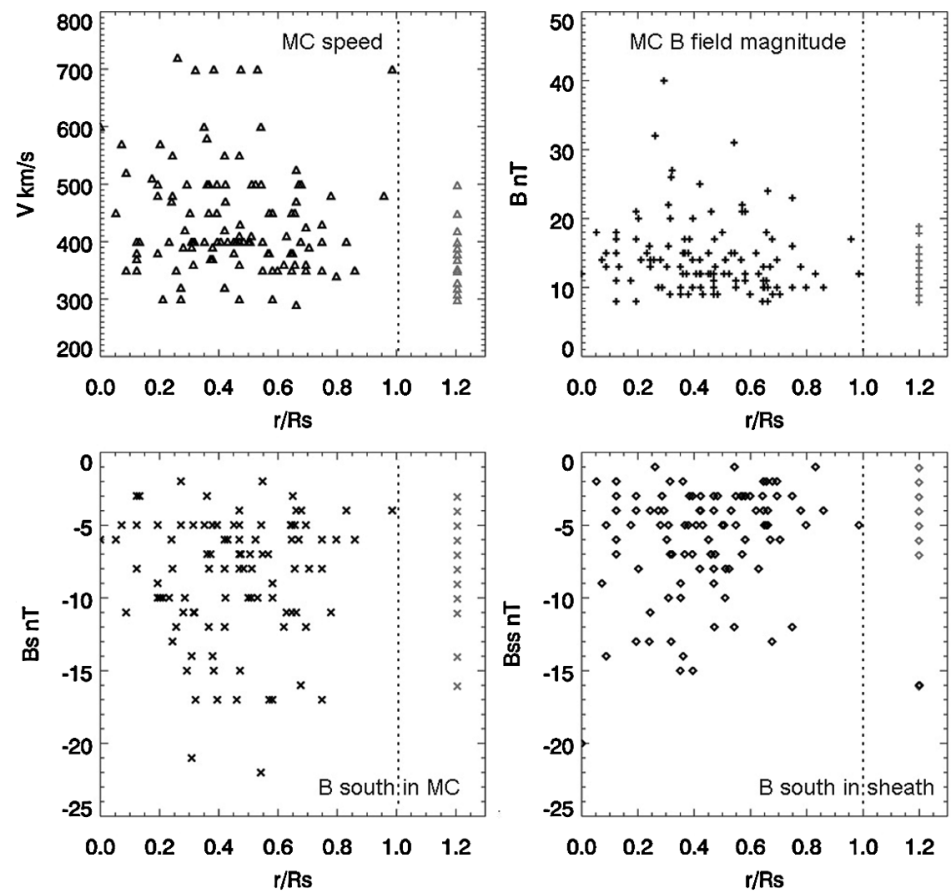

Figure 7 Scatter plots of MC parameters and normalized distance to solar disk center $\left[r / \mathrm{R}_{\odot}\right.$ : see text for definition]. (a) Maximum MC speed [ $V] v s . r / \mathrm{R}_{\odot}$; (b) maximum MC $B$ field magnitude $v s . r / \mathrm{R}_{\odot} ;$ (c) maximum MC $B$ south $\left[B_{\mathrm{S}}\right] v s . r / \mathrm{R}_{\odot}$; and (d) maximum sheath $B$ south $\left[B_{\mathrm{ss}}\right] v s . r / \mathrm{R}_{\odot}$.

where the bar plot indicates that the polarities of the resulting bipolar MCs have a clear solar-cycle dependence.

Next, we investigate whether there is any association between MC parameters at the Earth and their solar source location. The MC speed and magnetic-field strength, particularly the $B$ south, are of the greatest concern for space weather. In Figure 7, we present four scatter plots of MC parameters with normalized distance to the solar disk center $\left[r / \mathrm{R}_{\odot}\right]$, where $r$ is the distance of an MC source to solar disk center and is defined using source latitude $[\theta]$ and longitude $[\varphi]$ in solar coordinates:

$$
r / R_{\odot}=\sqrt{\cos ^{2} \theta \sin ^{2} \varphi+\sin ^{2} \theta} .
$$

For MCs of unclear sources, we assign $r / \mathrm{R}_{\odot}=1.2$ arbitrarily for display. Figure $7 \mathrm{a}$ shows the scatter of MC maximum speed [V] versus $r / \mathrm{R}_{\odot}$ in black-triangle symbols, and the vertically lined up gray triangles are the speed of MCs of unclear sources, which are all less than around $500 \mathrm{~km} \mathrm{~s}^{-1}$. It appears that all MCs faster than $600 \mathrm{~km} \mathrm{~s}^{-1}$ have sources within $r / \mathrm{R}_{\odot}<0.6$, except for one outlier. Figure $7 \mathrm{~b}$ shows the scatter of MC maximum magneticfield magnitude $[B]$ versus $r / R_{\odot}$ as black plus symbols, and the gray plus symbols are for MCs of unclear sources. MCs of maximum $B$ magnitude stronger than $25 \mathrm{nT}$ appear to have sources within $r / \mathrm{R}_{\odot}<0.6$ without exceptions. MCs of unclear sources all have a maximum $B$ magnitude below $20 \mathrm{nT}$. Figure 7c shows the scatter of the MC maximum southward magnetic field $\left[B_{\mathrm{s}}\right]$ versus $r / \mathrm{R}_{\odot}$ in black-cross symbols, and the gray-cross symbols are for MCs of unclear sources. $B_{\mathrm{s}}$ appears to be less ordered by the MCs' source location than $B$ 
magnitude, but it does also show a weak tendency of having stronger values with sources closer to solar disk center. In only one MC is $B_{\mathrm{s}}$ stronger than $-20 \mathrm{nT}$ in Solar Cycle 24 by the end of year 2017. Figure $7 \mathrm{~d}$ shows the scatter of the southward magnetic field in MC sheath $\left[B_{\mathrm{ss}}\right]$ versus $r / \mathrm{R}_{\odot}$ in black-diamond symbols, and the gray diamonds are for MCs of unclear sources. $B_{\mathrm{ss}}$ shows a noticeable trend of being stronger when MC sources lie closer to the solar disk center. The values of $B_{\mathrm{ss}}$ for MCs with unclear sources are all below $8 \mathrm{nT}$ with one exception.

\section{Geomagnetic Impacts of Magnetic Clouds}

Geomagnetic storms can be measured by the Dst index, and larger depressions of the Dst index indicate stronger storms. The Dst index is particularly sensitive to the IMF south Bz field and the solar-wind dynamic pressure (e.g. Kilpua et al., 2014). In Solar Cycle 23 (1996 to 2008), there were 17 very large storms of Dst $<-200 \mathrm{nT}$. We used (hourly) Dst values in the Omni2 data at the OMNIWeb NASA GSFC. Out of the 17 storms, 14 storms (82.4\%) were caused by MC events. Out of these 14 storms, the corresponding MCs and their sheath regions both had south $\mathrm{Bz}$ in ten events, one event had south $\mathrm{Bz}$ in only the $\mathrm{MCs}$, and three events had south Bz in only the sheath regions. The current Solar Cycle 24 (2009 to 2017) had 19 large storms of Dst $<-100 \mathrm{nT}$ (including only 2 very large geomagnetic storms of Dst $<-200 \mathrm{nT}$ ). Out of the 19 large storms, 14 storms (73.7\%) are caused by MC events. Out of the 14 storms, the corresponding MCs and their sheath regions both have south $\mathrm{Bz}$ in 11 events, 2 events have south $\mathrm{Bz}$ in only the MCs, and 1 event has south $\mathrm{Bz}$ in only the sheath region.

The three largest geomagnetic storms in Cycle 24 have minimum Dst values of $-220 \mathrm{nT}$, $-201 \mathrm{nT}$, and $-165 \mathrm{nT}$. The sources of the largest and the third largest storms are MCs, which we will show as examples. Figure 8 shows the solar wind, IMF observations, and the associated Dst response for the two MC-driven storm intervals in Cycle 24. In Figure 8a we see the MC (shaded in magenta) starting on 17 March 2015 and the corresponding Dst time series for the largest storm. This is a SN MC; the front part of the MC has a smooth, southward $\mathrm{Bz}$ field lasting $\approx 15$ hours with the minimum at $\approx-22 \mathrm{nT}$, and the $\mathrm{MC}$ speed is $\approx 600 \mathrm{~km} \mathrm{~s}^{-1}$. This MC has the strongest $B$ south in the current cycle, but it is also moderately fast. The combination of the $B_{\mathrm{s}}$ in the $\mathrm{MC}$ and the $B_{\mathrm{ss}}$ in its sheath region (shaded in pale yellow) right before it caused the largest geomagnetic storm of the current solar cycle. The Dst in the bottom row shows that the initial depression corresponds to the sheath region ahead of the $\mathrm{MC}$, where a south $\mathrm{Bz}$ field of minimum $\approx-12 \mathrm{nT}$ is present, at the same time, the solar-wind density in the sheath is higher than that within the MC by a factor $\approx 5$, which produces a larger dynamic pressure. The MC sheath caused the first stage of the storm, producing a Dst $\approx-70 \mathrm{nT}$, and the MC continues to cause the second stage of the storm. In Figure 8b, we show an MC (shaded in magenta) starting on 20 December 2015 and the corresponding Dst time series for the third largest storm. This is also a SN MC, the south $\mathrm{Bz}$ in the MC lasted $\approx 24$ hours with a minimum of $\approx-17 \mathrm{nT}$, and the speed is $\approx 400 \mathrm{~km} \mathrm{~s}^{-1}$. The sheath region (shaded in pale yellow) ahead of the MC has south $\mathrm{Bz}$ of $\approx-15 \mathrm{nT}$ at the peak, a higher speed of $\approx 500 \mathrm{~km} \mathrm{~s}^{-1}$ and a solar-wind density over $\approx 10$ times greater than that within the MC. The corresponding Dst curve again shows that the combination of the sheath and MC driver caused this two-stage strong storm.

The south $\mathrm{Bz}$ in MCs and in their sheath regions are both important in causing spaceweather events and need to be taken into account in related studies and forecasting. 

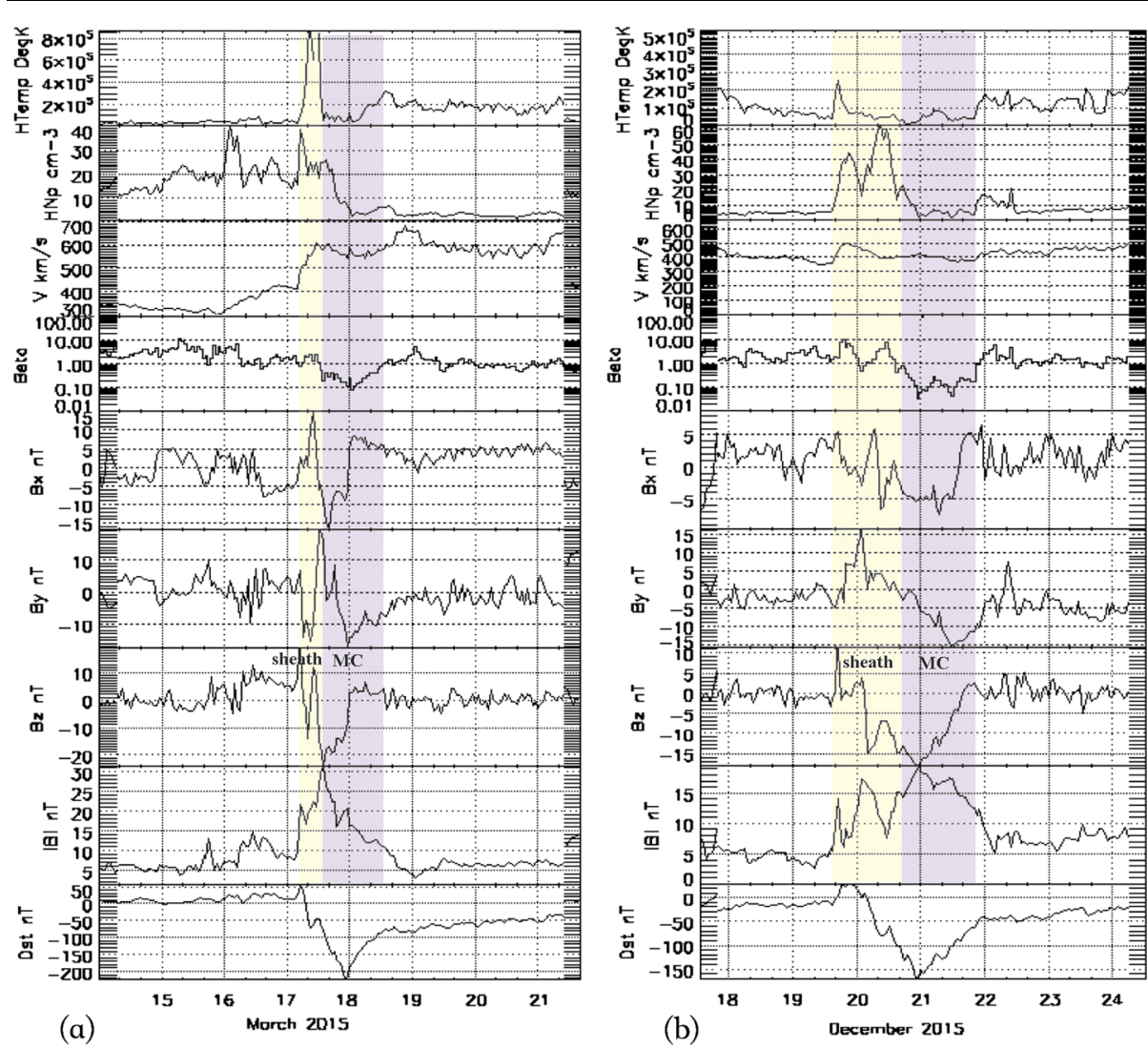

Figure 8 Solar-wind and IMF measurements and Dst index of two time intervals of the strongest Dst magnetic storms. From top to bottom, we show the solar-wind proton temperature, density, bulk speed, plasma $\beta$, and the IMF Bx, By, Bz, and B magnitude; the bottom row shows the Dst index. (a) Event in March 2015, (b) event in December 2015; the sheath regions and MCs are shaded in pale yellow and magenta, respectively.

During the current solar cycle, the majority of bipolar MCs have north-first field (NS $\mathrm{MCs}$ ), while two of the three largest geomagnetic storms are due to two south-first MCs (SN $\mathrm{MCs}$ ) combined with the southward field and large dynamic pressure in their sheath regions ahead of the MCs. We recall Figure 2 in Section 4, for the SN MCs in red-circle symbols and S MCs in black-cross symbols in Figure 2a. If south fields in their sheath regions $\left[B_{\mathrm{ss}}\right]$ as seen in the red- and black-square symbols in Figure $2 d$ have high values, dual actions of the two parts of south $B$ fields in close succession have a good chance of causing large two-stage Dst storms. It is interesting to note that the green squares in Figure $2 \mathrm{~d}$ are for $\mathrm{Bz}$ south fields in the sheath regions ahead of unipolar N MCs. In these cases, the sheath $\mathrm{Bz}$ south fields are the only geoeffective part of the events because the internal fields of N MCs produce little Dst response.

\section{Long-Term Solar-Cycle Dependence of Bipolar MCs}

It has been shown that in bipolar MCs, the North-South component (Bz) reverses with the same periodicity as the solar magnetic field (Li et al., 2011, 2014). Li et al. (2014) reported 


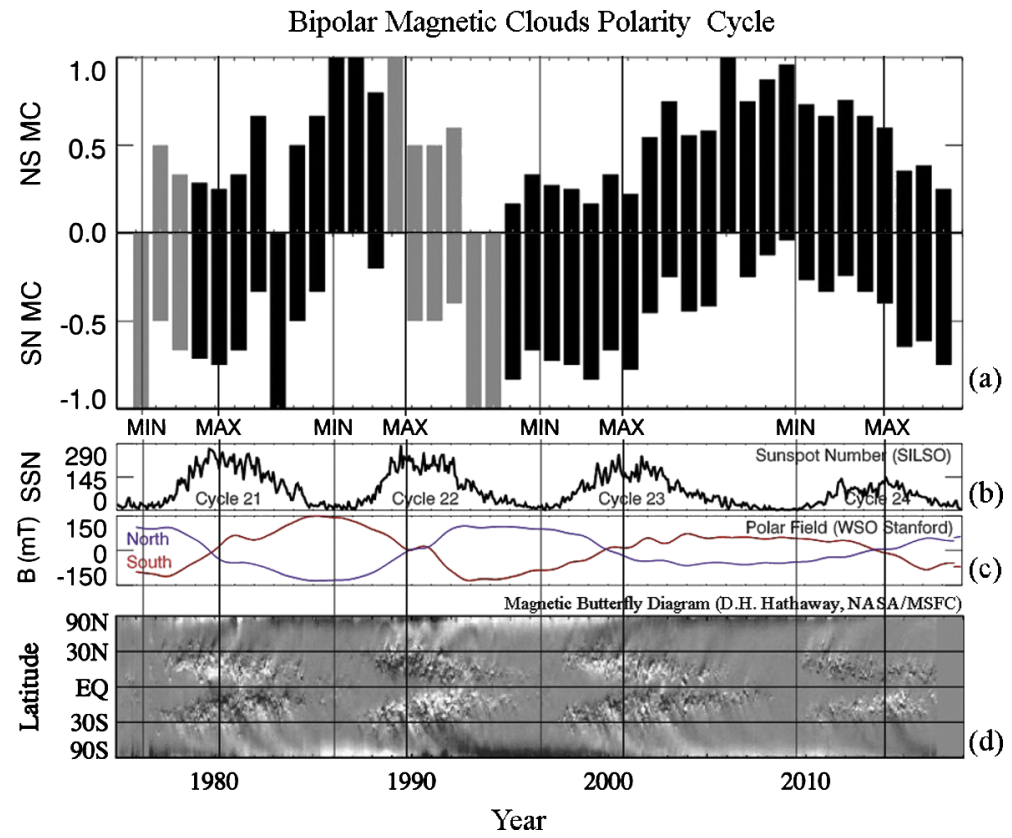

Figure 9 (a) Bipolar MC field solar-cycle dependence spanning four solar cycles, or 42 years, from 1976 to 2017. The gray bars indicate where the data are sparse with frequent and long data gaps, and therefore the MC counts are not as reliable. (b) Sunspot numbers of four solar cycles; (c) solar polar magnetic field of four solar cycles; (d) solar magnetic butterfly diagram by D. Hathaway (NASA).

the solar-cycle dependence of the MC field polarity by combining data from 1976 to 2012. In the current study, we extend the MC polarity long-term variation through to 2017 . The extended results, normalized as described below to better illustrate cycle phase relationships, are shown in Figure 9a (the previous study showed straight annual counts at each observing point). Here, a simple normalization has been applied to the number of NS (or SN) MCs as $f=n / n_{\mathrm{t}}$, where $n$ is the number of NS (or SN) MCs in a year, and $n_{\mathrm{t}}$ is the total number of the bipolar MCs of the same year. Figure 9 also provides the reference parameters of the solar cycle, including the sunspot numbers (b), the solar polar magnetic field (c), and the solar magnetic butterfly diagram up to 2016 (d) made available by D. Hathaway (NASA). The gray bars in Figure 9a indicate where the data are sparse with frequent and long data gaps, and therefore the $\mathrm{MC}$ counts are not as reliable.

The MC polarity on the rising phase of the solar cycle between a solar minimum (MIN) and the next solar maximum (MAX) usually are clearly dominated by either NS or SN MCs. Between 1986 (MIN) and 1990 (MAX) MCs are mostly of the NS type, between 1996 (MIN) and 2000 (MAX) MCs are mostly of the SN type, and between 2009 (MIN) and 2014 (MAX), MCs are again back to mostly NS type. We note that during some years around a solar minimum, MCs are always almost pure NS or SN with little exception. The dominant polarity reverses on the declining phase between the solar maximum and the next minimum. The last sunspot minimum and maximum occurred in 2009 and 2014, respectively. The Sun is currently in the declining phase of its activity cycle. The bipolar MC polarity in 2013 to 2017 shows large fluctuations between the numbers of the two types of bipolar MCs, while the overall polarity reversal of switching from a majority of NS MCs to having a majority of SN MCs is evident. 


\section{Discussion and Conclusions}

Detailed analyses of modern era solar-wind and IMF data from 1995 to 2017, spanning the recent two Solar Cycles 23 and 24, have been carried out. We have studied both bipolar and unipolar MCs. In addition to their occurrences and orientation polarities, we investigated the solar-cycle and source-region dependence of the MC events' maximum speed, magnetic magnitude, $\mathrm{Bz}$ south component within the MCs, and in the sheath regions ahead of the MCs. The occurrence of bipolar MCs has a less clear solar-cycle dependence, while their polarity does have a clear dependence on the solar cycle. Unipolar MCs mostly occur around solar active times, and their polarity does not depend on the solar cycle. The solarcycle dependence of the polarity of bipolar MCs has been known and generally accepted for decades. With the new data of recent solar cycles, we now make a quantitative analysis of this dependence and the dependence of MC occurrence on the solar cycle. Figures 10a, b, and c give the overplots of the bipolar MC polarity with solar north (blue) and south (red) polar field values, and the bipolar and unipolar MC occurrences with sunspot numbers; and Figures 10d, e, and f show their scatter plots and the results of their linear fits. Figure 10d presents the bipolar MC polarity versus the solar polar-field strength, and the Pearson linear correlation coefficient $[R]$ is $\approx 0.70$. The Spearman rank correlation coefficients $[\rho]$ give similar values of $\approx 0.70$ with a decent significance of the $\mathrm{p}$-value $\lesssim 10^{4}$. These analyses confirm a quite strong correlation of the polarity on the solar cycle. Figure 10e shows the bipolar MC occurrence versus sunspot numbers. The Pearson correlation is $\approx 0.4$ and the rank correlation is 0.50 , with much less significant $\mathrm{p}$-values of $\approx 0.01-0.02$. The dependence of the bipolar MC occurrence on solar cycle is statistically much weaker. Figure $10 \mathrm{f}$ gives the unipolar MC occurrence versus sunspot numbers. The Pearson correlation is $\approx 0.7$ and the rank correlation is $\approx 0.7$, with a decent significance of the $\mathrm{p}$-value $\lesssim 10^{-4}$, similar to the polarity results and thus a clear solar-cycle dependence.

Eighty-five percent of a total of 303 MCs during the recent two solar cycles contained a south $\mathrm{Bz}$ component for some part or for the entire duration of their passage over the Earth, highlighting the space-weather importance of MCs. The MCs with the highest speed, strongest magnetic magnitude, and largest Bz south component occur in the declining phase of the solar cycle, from the solar maximum to one or two years before the next solar minimum. When bipolar MCs are grouped by the type of their solar source, the polarity of MCs from active-region flares does not show a solar-cycle dependence, while those from large filament sources and uncertain sources show a similar solar-cycle dependence. Based on these new findings, the solar-cycle dependence of the polarity of bipolar MCs is mostly from the contributions of quiescent filament eruptions and some weak events of uncertain sources (including stealth CMEs), and the fluctuations or outliers in the picture are mostly from active-region flare-associated MC events.

Active-region magnetic fields are stronger and can have considerably more complexity, variation, and evolution of their flux distributions and in their orientations than the surrounding corona (Ugarte-Urra, Warren, and Winebarger, 2007). Large, quiescent filaments are from decayed magnetic regions whose on-disk polarity often agrees with the large-scale magnetic field of the corona ( $\mathrm{Li}$ and Luhmann, 2006). A part of the MC events from uncertain sources are identified as stealth CMEs, which originate from greater heights in the corona and involve the gradual eruption of larger-scale helmet streamer fields. The global helmet-streamer belt fields are weaker, less complex, and typically reflect the orientation of the global solar dipole. CMEs from strong, active-region magnetic structures may rotate more after eruptions due to their stronger internal stress (e.g. Lynch et al., 2009) in addition to their highly variable active-region source and flux-rope orientations (Leamon, Canfield, 

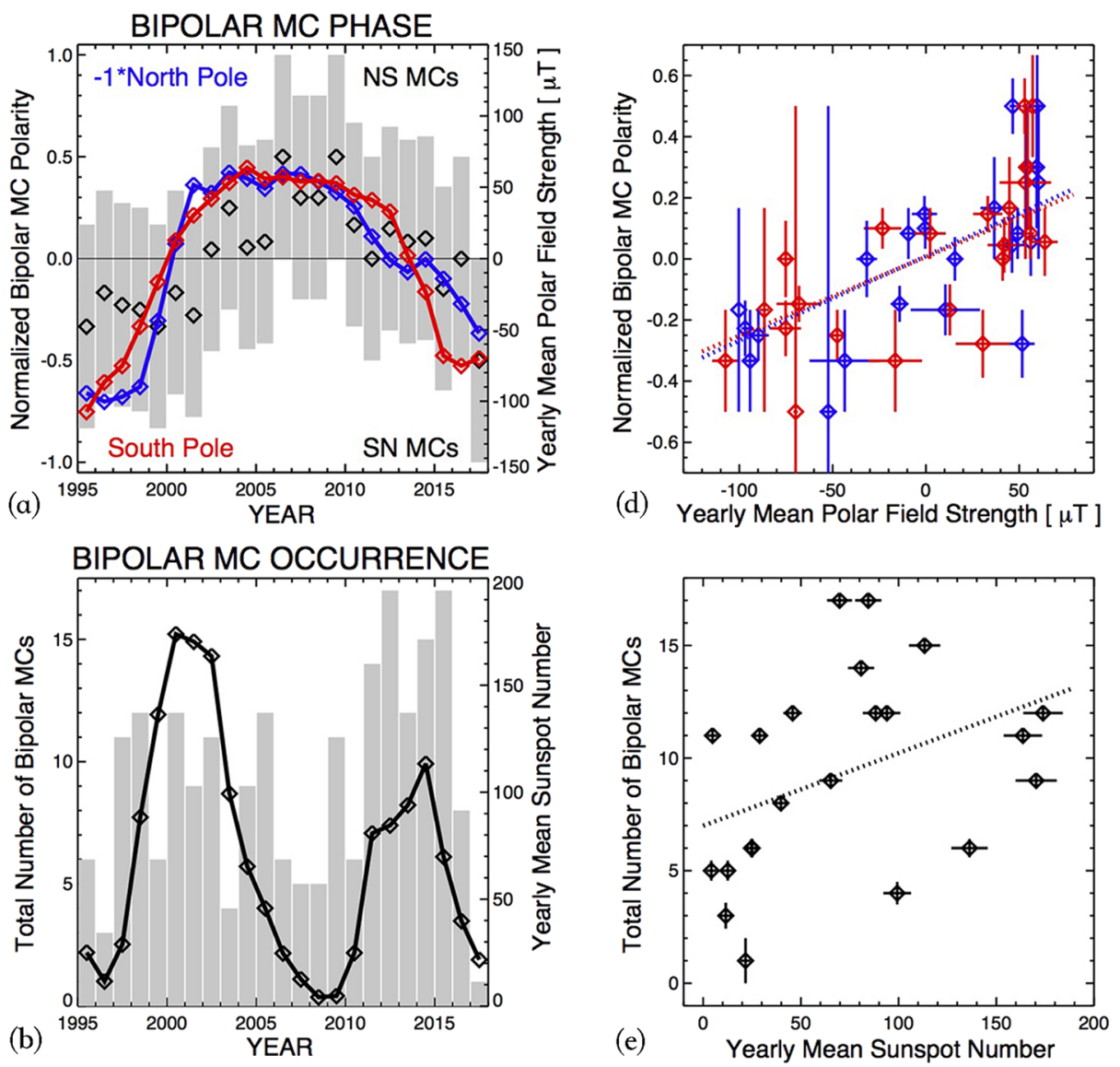

\section{UNIPOLAR MC OCCURRENCE}
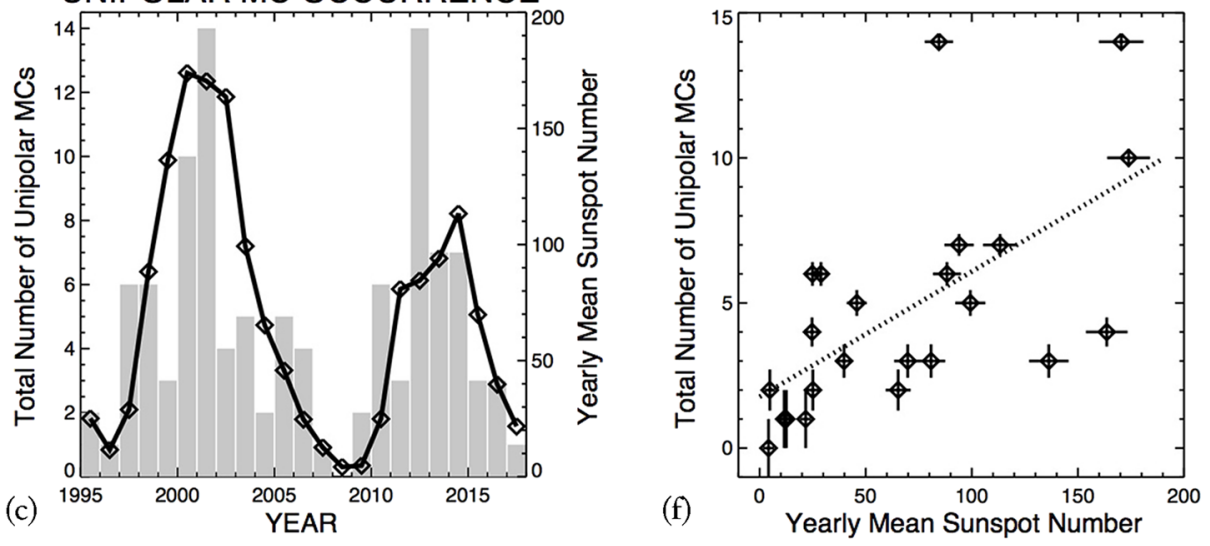

Figure 10 Statistical analyses of MC parameters vs. solar-cycle index. (a) Solar polar-field strength over bipolar MC polarity phase, blue for the north polar field multiplied by -1 and red for the south polar field, and black diamonds are the midpoint of each bars representing normalized annual counts of NS and SN bipolar MCs. (b) Sunspot numbers over bipolar MC occurrence. (c) Sunspot numbers over unipolar MC occurrence. (d), (e), (f) Scatter plot and linear fit of the MC parameter and solar-cycle index corresponding to the panel on the left, respectively. 
and Pevtsov, 2002; Leamon et al., 2004). Therefore the magnetic-field orientations of MCs from active regions are expected to be less predictable in a general sense. Unfortunately, this group of events poses the most threat to space weather because they are the source-region category that usually produces the most energetic eruptions resulting in the strongest $\mathrm{MC}$ magnetic fields and fastest MC speeds. A better understanding of the detailed processes of CME eruptions from different sources and their heliospheric propagation is needed for successful space-weather forecasting.

The source regions of Earth-impacting MCs are located within $45^{\circ}$ of the solar disk center with a few exceptions. On average, the filament sources tend to be located at higher latitudes than flare sources. We find that the MCs with the highest speeds, largest total $B$ magnitudes, and sheath $\mathrm{Bz}$ south are from source regions closer to the solar disk center, within about $30^{\circ}$ latitude and longitude. The velocity and field parameters of MCs with unclear sources are all generally low and small. While this type of CME may be the most difficult to predict, statistically, these events should not have severe space-weather impacts. Our MC database is inclusive of all of the in-situ MC events observed at 1 AU in the past 11 years: 2007-2017. The combination of the Bz south fields in the sheath regions and SN MCs are the cause of the largest geomagnetic storms. In addition, these cases show that storms can be given only by the south $\mathrm{Bz}$ in the sheath regions. Thus, for space-weather forecasting, the magnetic field in the sheath regions of MCs should also be taken into consideration in addition to the field within MCs.

We updated the record of the solar-cycle dependence of bipolar magnetic clouds (MCs) using newly available solar-wind and IMF data obtained near $1 \mathrm{AU}$ and the ecliptic plane. The MC polarity trend, presented in a normalized format to emphasize solar-cycle phase information, now spans about four solar cycles (42 years), from 1976 to 2017. The MC polarity in the rising phase of the solar cycle is clearly dominated by one type of bipolar MC, either NS or SN. During a few years around a solar minimum, MCs are always almost pure NS or SN with little exception. Mixed polarities begin to appear with the increase of solar activity and approaching the solar maximum. The predominant MC polarity reverses within the declining phase. The last sunspot minimum and maximum occurred in 2009 and 2014, respectively. The Sun is currently in the declining phase of Cycle 24. The bipolar MC polarity in 2013 to 2017 shows large fluctuations between the occurrences of the two types of bipolar MCs while maintaining the phase of the cyclic polarity reversal switching from NS MCs as the major type to SN MCs as the major type.

The implications of ICMEs in the solar magnetic cycle have been conceptually considered in the past as part of the picture of the solar-dynamo operation (e.g. Käpylä, Korpi, and Brandenburg, 2010; Warnecke and Brandenburg, 2010, 2014; Warnecke, Brandenburg, and Mitra, 2011; Warnecke et al., 2012). Although this topic is not the focus of this article, our finding that quiescent filament CMEs and stealth CMEs give rise to the MC polarity cycle suggests that these MCs participate in removing the like polarity of the solar dipole field. MCs that are related to flares, on the other hand, likely play a role in removing and relaxing the toroidal fields of solar active regions. In this respect, the phases of the changes in the MC polarities compared to the sunspot-number and polar-field-polarity cycles (see Figure 9) are key observational results. Care must be taken, however, to take into account the observational biases represented by the near-ecliptic observations at 1 AU. Additional polarity analyses performed on Ulysses high-latitude ICMEs, as well as the upcoming Solar Orbiter ICMEs, should prove interesting and complementary in this regard.

Acknowledgements This work was supported in part by NASA Grant NNX15AG09G to the University of California, Berkeley for the STEREO/IMPACT investigation. Y. Li acknowledges the support of NASA LWS grant NNX15AB80G. Y. Li and B.J. Lynch are supported by NSF grant SHINE-1622495 and NASA 
HSR grant NNX17AI28G. B.J. Lynch is also supported by the Coronal Global Evolutionary Model (CGEM) project NSF AGS-1321474.

We acknowledge ACE/MAG and /SWEPAM level-2 data and OMNI2 data, STEREO/ IMPACT and /SECCHI data, and SDO/AIA imaging data. We acknowledge the LASCO CME catalog generated and maintained at the CDAW Data Center by NASA and The Catholic University of America in cooperation with the Naval Research Laboratory. SOHO is a project of international cooperation between ESA and NASA.

Disclosure of Potential Conflicts of Interest The authors declare that they have no conflicts of interest.

Open Access This article is distributed under the terms of the Creative Commons Attribution 4.0 International License (http://creativecommons.org/licenses/by/4.0/), which permits unrestricted use, distribution, and reproduction in any medium, provided you give appropriate credit to the original author(s) and the source, provide a link to the Creative Commons license, and indicate if changes were made.

\section{References}

Alzate, N., Morgan, H.: 2017, Identification of low coronal sources of "stealth" coronal mass ejections using new image processing techniques. Astrophys. J. 840, 103. DOI.

Bothmer, V., Rust, D.M.: 1997, The field configuration of magnetic clouds and the solar cycle. In: Crooker, N., Joselyn, J.A., Feynman, J. (eds.) Coronal Mass Ejections, Geophys. Monogr. Ser. 99, AGU, Washington, 139. DOI.

Bothmer, V., Schwenn, R.: 1998, The structure and origin of magnetic clouds in the solar wind. Ann. Geophys. 16, 1. DOI.

Burlaga, L.F.: 1988, Magnetic clouds and force-free fields with constant alpha. J. Geophys. Res. Space Phys. 93, 7217. DOI.

Chi, Y., Shen, C., Wang, Y., Xu, M., Ye, P., Wang, S.: 2016, Statistical study of the interplanetary coronal mass ejections from 1996 to 2015. Solar Phys. 291, 2419. DOI.

D’Huys, E., Seaton, D.B., Poedts, S., Berghmans, D.: 2014, Observational characteristics of coronal mass ejections without low-coronal signatures. Astrophys. J. 795, 49. DOI.

Gopalswamy, N., Akiyama, S., Yashiro, S., Michalek, G., Lepping, R.P.: 2008, Solar sources and geospace consequences of interplanetary magnetic clouds observed during solar cycle 23. J. Atmos. Solar-Terr. Phys. 70, 245. DOI.

Hess, P., Zhang, J.: 2017, A study of the Earth-affecting CMEs of Solar Cycle 24. Solar Phys. 292, 80. DOI.

Jian, L.K., Russell, C.T., Luhmann, J.G.: 2011, Comparing solar minimum 23/24 with historical solar wind records at 1 AU. Solar Phys. 274, 321. DOI.

Jian, L.K., Russell, C.T., Luhmann, J.G., Skoug, R.M.: 2006, Properties of interplanetary coronal mass ejections at one AU during 1995 - 2004. Solar Phys. 239, 393. DOI.

Jian, L.K., Russell, C.T., Luhmann, J.G., Galvin, A.B., Simunac, K.D.C.: 2013, Solar wind observations at STEREO: 2007-2011. In: SOLAR WIND 13: Proc. Thirteenth International Solar Wind Conf., CP1539, 195, AIP, Melville. DOI.

Käpylä, P.J., Korpi, M.J., Brandenburg, A.: 2010, Open and closed boundaries in large-scale convective dynamos. Astron. Astrophys. 518, A22. DOI.

Kilpua, E.K.J., Luhmann, J.G., Jian, L.K., Russell, C.T., Li, Y.: 2014, Why have geomagnetic storms been so weak during the recent solar minimum and the rising phase of cycle 24? J. Atmos. Solar-Terr. Phys. 107, 12. DOI.

Krista, L.D., Reinard, A.A.: 2017, Statistical study of solar dimmings using CoDiT. Astrophys. J. 839, 50. DOI.

Leamon, R.J., Canfield, R.C., Pevtsov, A.A.: 2002, Properties of magnetic clouds and geomagnetic storms associated with eruption of coronal sigmoids. J. Geophys. Res. 107, 1234. DOI.

Leamon, R.J., Canfield, R.C., Jones, S.L., Lambkin, K., Lundberg, B.J., Pevtsov, A.A.: 2004, Helicity of magnetic clouds and their associated active regions. J. Geophys. Res. 109, A05106. DOI.

Lee, C.O., Luhmann, J.G., Zhao, X.P., Liu, Y., Riley, P., Arge, C.N., Russell, C.T., de Pater, J.: 2009, Effects of the weak polar fields of Solar Cycle 23: investigation using OMNI for the STEREO mission period. Solar Phys. 256, 345. DOI.

Li, Y., Luhmann, J.G.: 2006, Coronal magnetic field topology over filament channels: implication for coronal mass ejection initiations. Astrophys. J. 648, 732. DOI.

Li, Y., Luhmann, J.G., Lynch, B.J., Kilpua, E.K.J.: 2011, Cyclic reversal of magnetic cloud poloidal field. Solar Phys. 270, 331. DOI. 
Li, Y., Luhmann, J.G., Lynch, B.J., Kilpua, E.K.J.: 2014, Magnetic clouds and origins in STEREO era. J. Geophys. Res. Space Phys. 119, 3237. DOI.

Lugaz, N., Temmer, M., Wang, Y., Farrugia, C.J.: 2017, The interaction of successive coronal mass ejections: a review. Solar Phys. 292, 64. DOI.

Lynch, B.J., Antiochos, S.K., Li, Y., Luhmann, J.G., DeVore, C.R.: 2009, Rotation of coronal mass ejections during eruption. Astrophys. J. 697, 1918. DOI.

Lynch, B.J., Masson, S., Li, Y., DeVore, C.R., Luhmann, J.G., Antiochos, S.K., Fisher, G.H.: 2016, A model for stealth coronal mass ejections. J. Geophys. Res. Space Phys. 121, 10,677. DOI.

Mulligan, T., Russell, C.T., Luhmann, J.G.: 1998, Solar cycle evolution of the structure of magnetic clouds in the inner heliosphere. Geophys. Res. Lett. 25, 2959. DOI.

Richardson, I.G., Cane, H.V.: 2010, Near-Earth interplanetary coronal mass ejections during Solar Cycle 23 (1996 - 2009): catalog and summary of properties. Solar Phys. 264, 189. DOI.

Richardson, I.G., Cane, H.V.: 2013, Solar wind drivers of geomagnetic storms over more than four solar cycles. In: Zank, G.P., Borovsky, J., Bruno, R., Cirtain, J., Cranmer, S., Elliott, H., Giacalone, J., Gonzalez, W., Li, G., Marsch, E., Moebius, E., Pogorelov, N., Spann, J., Verkhoglyadova, O. (eds.) SOLAR WIND 13: Proc. Thirteenth International Solar Wind Conf. CP-1539, AIP, Melville, 422. DOI.

Riley, P.: 2016, Predicting Bz: Baby Steps. Am. Geophys. Union, Fall Gen. Assemb. Abstract \#SM23C-01.

Riley, P., Love, J.J.: 2017, Extreme geomagnetic storms: probabilistic forecasts and their uncertainties. Space Weather 15, 53. DOI.

Robbrecht, E., Patsourakos, S., Vourlidas, A.: 2009, No trace left behind: STEREO observation of a coronal mass ejection without low coronal signatures. Astrophys. J. 701, 283. DOI.

Thompson, B.J., Plunkett, S.P., Gurman, J.B., Newmark, J.S., St. Cyr, O.C., Michels, D.J.: 1998, SOHO/EIT observations of an Earth-directed coronal mass ejection on May 12, 1997. Geophys. Res. Lett. 25, 2465. DOI.

Ugarte-Urra, I., Warren, H.P., Winebarger, A.R.: 2007, The magnetic topology of coronal mass ejection sources. Astrophys. J. 662, 1293. DOI.

Warnecke, J., Brandenburg, A.: 2010, Surface appearance of dynamo-generated large-scale fields. Astron. Astrophys. 523, A19. DOI.

Warnecke, J., Brandenburg, A.: 2014, Coronal influence on dynamos. In: Magnetic Fields Throughout Stellar Evolution, Proc. Internat. Astron. Union, Symp. 302, 134. DOI.

Warnecke, J., Brandenburg, A., Mitra, D.: 2011, Plasmoid ejections driven by dynamo action underneath a spherical surface. In: Bonanno, A., de Gouveia Dal Pino, E., Kosovichev, A.G. (eds.) Advances in Plasma Astrophysics, Proc. Internat. Astron. Un. Symp. 274, Cambridge Univ. Press, Cambridge, 306. DOI.

Warnecke, J., Käpylä, P.J., Mantere, M.J., Brandenburg, A.: 2012, Coronal ejections from convective spherical shell dynamos. In: Mandrini, C.H., Webb, D.F. (eds.) Comparative Magnetic Minima: Characterizing quiet times in the Sun and Stars, Proc. Internat. Astron. Un. Symp. 286, Cambridge Univ. Press, Cambridge, 154. DOI.

Zhang, G., Burlaga, L.F.: 1988, Magnetic clouds, geomagnetic disturbances, and cosmic ray decreases. J. Geophys. Res. Space Phys. 93, 2511. DOI.

Zhang, J., Liemohn, M.W., Kozyra, J.U., Lynch, B.J., Zurbuchen, T.H.: 2004, A statistical study of the geoeffectiveness of magnetic clouds during high solar activity years. J. Geophys. Res. Space Phys. 109, A09101. DOI.

Zhang, J., Richardson, I.G., Webb, D.F., Gopalswamy, N., Huttunen, E., Kasper, J.C., Nitta, N.V., et al.: 2007, Solar and interplanetary sources of major geomagnetic storms (Dst $\leq-100 \mathrm{nT}$ ) during $1996-$ 2005. J. Geophys. Res. Space Phys. 112, A10102. DOI. 